\title{
A COCYCLE ON THE GROUP OF SYMPLECTIC DIFFEOMORPHISMS
}

\author{
ŚWIATOSŁAW R. GAL AND JAREK KĘDRA
}

\begin{abstract}
Авstract. We define a cocycle on the group of Hamiltonian diffeomorphisms of a symplectically aspherical manifold and investigate its properties. The main application is an alternative proof of the Polterovich theorem about the distortion of cyclic subgroups in finitely generated groups of Hamiltonian diffeomorphisms.
\end{abstract}

\section{Contents}

1. Introduction

2. A cocycle on $\operatorname{Symp}(M, \omega=d \alpha)$.

3. The Polterovich homomorphism

4. The non-triviality of $\mathcal{K}$

5. Distortion of cyclic subgroups

6. A proof of the Polterovich theorem

7. The relation with the flux homomorphism References

\section{INTRODUCTION}

In the present paper we investigate the properties of a certain cocycle defined on the group of Hamiltonian diffeomorphisms of a symplectically aspherical manifold. The cocycle appears more or less explicitly in Gambaudo-Ghys [5] and Arnold-Khesin [1, p. 247] for the case of a symplectic ball.

As an application we proof a theorem due to Polterovich [17] that a finitely generated subgroup of the group of Hamiltonian diffeomorphisms of a symplectically hyperbolic manifold has undistorted cyclic subgroups.

Date: September 19, 2021.

1991 Mathematics Subject Classification. Primary 57S25; Secondary 20 F69.

Key words and phrases. symplectic manifold; group action; discrete group. 
1.1. Distortion of cyclic subgroups. Let $\Gamma$ be a finitely generated group and let $|g|$ denote the word norm of $g \in \Gamma$ with respect to some fixed finite set of generators. A cyclic subgroup $\langle g\rangle \subset \Gamma$ generated by $g$ is called undistorted if there exists a positive constant $C$ such that for any integer $n \in \mathbb{Z}$ there holds $\left|g^{n}\right| \geq C \cdot|n|$.

Remark 1.2. According to Gromov's definition [8] finite subgroup are also undistorted. It follows from our definition that an undistorted cyclic subgroup is infinite.

Example 1.3 (Groups with undistorted cyclic subgroups). $\quad$ (1) Free and free Abelian groups.

(2) Hyperbolic groups.

(3) Groups acting properly discontinuously on contractible CAT(0)spaces.

Example 1.4 (Groups with distorted cyclic subgroups). (1) Groups with torsion.

(2) Heisenberg groups of the uppertriangular integer matrices.

(3) Non-uniform irreducible lattices in semisimple Lie groups (see Lubotzky-Mozes-Raghunathan [13]).

The first application is the following result which was also proved by Frauenfelder and Schlenk in [3, Theorem 3.7] by a different method.

Theorem 1.5. Let $(M, \omega)$ be an exact symplectic manifold convex at infinity. Let $h: \Gamma \hookrightarrow \operatorname{Ham}_{c}(M, \omega)$ be an effective Hamiltonian action of a finitely generated group. Then any non-trivial cyclic subgroup of $\Gamma$ is undistorted with respect to the word metric given by a finite set of generators.

Remark 1.6. It follows from the Thurston Stability (see for example Theorem 3.4 in Franks [2]) that a group acting smoothly with compact supports on an open manifold has many undistorted cyclic subgroups. We show that the reasons for the undistortion are not purely topological. Namely, in Section 5.4, we present an example, due to Łukasz Grabowski, of a smooth compactly supported action of the Heisenberg group on $\mathbb{R}^{n}$ for $n \geq 2$. It is, however, not clear if the above theorem holds for volume preserving actions.

A symplectic manifold is called exact if the symplectic form is exact. Notice that in this case $M$ is not closed. The boundary $\partial M$ of a symplectic manifold $(M, \omega)$ is called convex if there exists a vector field $X$ defined in a neighbourhood of $\partial M$ such that $\mathcal{L}_{X} \omega=\omega$ and it 
points outwards. A symplectic manifold $(M, \omega)$ is convex at infinity if there exists an increasing sequence of compact submanifolds with convex boundaries exhausting $M$.

Example 1.7 (Symplectic manifolds convex at infinity). (1) The standard symplectic Euclidean space $\left(\mathbb{R}^{2 n}, \omega\right)$.

(2) The cotangent bundle $\left(T^{*} X, d \lambda\right)$ over a smooth manifold $X$.

(3) Stein manifolds.

Roughly speaking our proof of Theorem 1.5 consists of the following steps. We define a cocycle $\mathcal{K}: \operatorname{Ham}_{c}(M, \omega) \rightarrow C_{c}^{\infty}(M)$ and compose it with a norm given by the oscillation. This induces a norm on the group $\operatorname{Ham}_{c}(M, \omega)$ and we prove that every nontrivial cyclic subgroup of $\operatorname{Ham}_{c}(M, \omega)$ is undistorted with respect to this norm. Finally we prove that the action $h: \Gamma \hookrightarrow \operatorname{Ham}_{\mathcal{C}}(M, \omega)$ is Lipschitz with respect to the word norm on $\Gamma$ and the above mentioned norm on $\operatorname{Ham}_{\mathcal{C}}(M, \omega)$. The proof is presented in Section 5.3.

Let $p: \widetilde{M} \rightarrow M$ be the universal covering. Making an observation (Proposition 2.12) that there exists a lifting homomorphism $\Psi: \operatorname{Ham}(M, \omega) \rightarrow \operatorname{Ham}\left(\widetilde{M}, p^{*} \omega\right)$, we extend the above argument to prove (in Section 6) the following result by Polterovich.

Theorem 1.8 (Polterovich, 1.6.C in [17]). Let $(M, \omega)$ be a closed symplectically hyperbolic manifold. Let $\Gamma \hookrightarrow \operatorname{Ham}(M, \omega)$ be an effective Hamiltonian action of a finitely generated group. Then $\Gamma$ has undistorted cyclic subgroups.

Both proofs rely on the existence of certain fixed points proved by Schwarz [18] for closed manifolds and Frauenfelder-Schlenk [4] for manifolds convex at infinity.

A symplectic manifold $(M, \omega)$ is said to be symplectically aspherical if the pull-back $p^{*} \omega$ of the symplectic form $\omega$ to the universal cover is exact, ie. $p^{*} \omega=d \alpha$ for some one-form $\alpha$. Observe that the symplectic asphericity is equivalent to the vanishing of the symplectic form on spheres. That is $\int_{S^{2}} f^{*} \omega=0$ for any smooth map $f: S^{2} \rightarrow M$. Clearly, the symplectic asphericity depends only on the cohomology class of the symplectic form.

Example 1.9 (Closed symplectically aspherical manifolds). Aspherical symplectic manifolds, such as tori, surfaces of positive genus and their products, are obviously symplectically aspherical. A branched covering of a symplectically aspherical manifold is again symplectically aspherical. This allows to produce more interesting examples possibly with non-trivial second and higher homotopy groups 
as it was done by Gompf in [6]. Also, a symplectic submanifold of a symplectically aspherical manifold is symplectically aspherical (see [9. 10] for other constructions and properties).

Let $(M, \omega)$ be a closed symplectic manifold equipped with a Riemannian metric $g$ and let $p: \widetilde{M} \rightarrow M$ be its universal cover. The manifold $(M, \omega)$ is called symplectically hyperbolic if it is symplectically aspherical and there exists a primitive $\alpha$ of $p^{*} \omega$ which is bounded with respect to the metric induced by $g$. That is there exists a constant $C \in \mathbb{R}$ such that

$$
\sup _{x \in \widetilde{M}}\|\alpha(x)\|<C,
$$

where $\|\alpha(x)\|:=\sup \left\{\alpha(x)(X) \mid X \in T_{x} M\right.$ and $\left.|X| \leq 1\right\}$.

Notice that the symplectic hyperbolicity does not depend on the choice of a Riemannian metric. Also, it depends only on the cohomology class of the symplectic form. Closed oriented surfaces of genus at least 2 and their products are symplectically hyperbolic as well as their branched covers and submanifolds. A symplectically aspherical manifold with word hyperbolic fundamental group is symplectically hyperbolic [11, Corollary 1.13].

Acknowledgements. We thank Michael Weiss and the University of Aberdeen for the support of the visit of the first author in Aberdeen in May 2008. The second author thanks Frédéric Bourgeois, Felix Schlenk and Thomas Vogel for discussions during the symplectic geometry seminar in Brussels in February 2008.

$$
\text { 2. A Cocycle on } \operatorname{Symp}(M, \omega=d \alpha) \text {. }
$$

2.1. Definition of the cocycle. Let $C^{\infty}(M)$ denote the vector space of real valued functions on $M$. The group $\operatorname{Symp}(M, \omega)$ acts on functions from the right by the composition. We assume that $M$ is connected throughout the paper.

Let $\alpha \in \Omega^{1}(M)$ be a primitive of the symplectic form $\omega$. That is $d \alpha=\omega$. Let $f \in \operatorname{Symp}(M, \omega)$ be a symplectic diffeomorphism. The one-form $f^{*} \alpha-\alpha$ is closed.

Proposition 2.2. Let $f \in \operatorname{Symp}_{0}(M, d \alpha)$. The form $f^{*}(\alpha)-\alpha$ is exact if and only if $f$ is Hamiltonian.

Proof. A diffeomorphism $f$ is Hamiltonian if and only if there exists a symplectic isotopy $f_{t}$ from the identity to $f$ such that

$$
\int_{f_{t}(\gamma)} d \alpha=0
$$


for any loop $\gamma: S^{1} \rightarrow M$ (McDuff-Salamon [15, Theorem 10.12]). Then we have that

$$
0=\int_{f_{t}(\gamma)} d \alpha=\int_{f(\gamma)-\gamma} \alpha=\int_{\gamma} f^{*} \alpha-\alpha
$$

which is equivalent to the exactness of $f^{*} \alpha-\alpha$.

Let $\widehat{\operatorname{Ham}}(M, d \alpha) \subset \operatorname{Symp}(M, d \alpha)$ be the kernel of the cocycle $\operatorname{Symp}(M, d \alpha) \rightarrow H^{1}(M ; \mathbb{R})$ defined by $f \mapsto\left[f^{*} \alpha-\alpha\right]$. Its connected component of the identity is the group of Hamiltonian diffeomorphisms. Clearly, if $H^{1}(M, R)=0$ then $\widehat{\operatorname{Ham}}(M, d \alpha)=\operatorname{Symp}(M, d \alpha)$. Enlargements of the group of Hamiltonian diffeomorphisms of a closed symplectic manifolds are investigated by McDuff in [14].

If $f \in \widehat{\operatorname{Ham}}(M, d \alpha)$ then there exists a function $\mathcal{K}_{\alpha}(f)$ such that $d \mathcal{K}_{\alpha}(f)=f^{*} \alpha-\alpha$. Such a function is unique up to a constant.

Proposition 2.3. Let $(M, d \alpha)$ be an exact symplectic manifold. The map $\mathcal{K}_{\alpha}: \widehat{\operatorname{Ham}}(M, \omega) \rightarrow C^{\infty}(M) / \mathbb{R}$ defined above is a cocycle.

Proof. We have to check that $\mathcal{K}_{\alpha}(f \circ g)=\mathcal{K}_{\alpha}(f) \circ g+\mathcal{K}_{\alpha}(g)$ up to a constant.

$$
\begin{aligned}
d \mathcal{K}_{\alpha}(f \circ g) & =(f \circ g)^{*} \alpha-\alpha \\
& =g^{*}\left(f^{*} \alpha\right)-g^{*} \alpha+g^{*} \alpha-\alpha \\
& =g^{*}\left(d \mathcal{K}_{\alpha}(f)\right)+d \mathcal{K}_{\alpha}(g) \\
& =d\left(\mathcal{K}_{\alpha}(f) \circ g\right)+d\left(\mathcal{K}_{\alpha}(g)\right)
\end{aligned}
$$

We get that $d\left(\mathcal{K}_{\alpha}(f \circ g)-\mathcal{K}_{\alpha}(f) \circ g-\mathcal{K}_{\alpha}(g)\right)=0$ which means that the function $\mathcal{K}_{\alpha}(f \circ g)-\mathcal{K}_{\alpha}(f) \circ g-\mathcal{K}_{\alpha}(g)$ is constant.

\subsection{Basic properties of $\mathcal{K}_{\alpha}$.}

Proposition 2.5. If $H^{1}(M ; \mathbb{R})=0$ then the cohomology class $\left[\mathcal{K}_{\alpha}\right] \in$ $H^{1}\left(\operatorname{Symp}(M, d \alpha) ; C^{\infty}(M) / \mathbb{R}\right)$ does not depend on the choice of a primitive.

Proof. Let $\beta \in \Omega^{1}(M)$ be another primitive of $\omega$. Then $\alpha-\beta=d F$. A straightforward calculation shows that $\mathcal{K}_{\alpha}(f)-\mathcal{K}_{\beta}(f)=F \circ f-$ $F$, which means that $\mathcal{K}_{\alpha}$ and $\mathcal{K}_{\beta}$ are cohomologous.

Proposition 2.6. There exists an injective homomorphism

$$
\iota: H^{1}(M ; \mathbb{R}) \rightarrow H^{1}\left(\operatorname{Ham}(M, d \alpha) ; C^{\infty}(M) / \mathbb{R}\right) .
$$

If $\alpha$ and $\beta$ are two primitives of the symplectic form then $\left[\mathcal{K}_{\alpha}\right]-\left[\mathcal{K}_{\beta}\right]=$ $\iota[\alpha-\beta]$. 
Proof. Let $f \in \operatorname{Ham}(M, d \alpha)$ and let $[a] \in H^{1}(M ; \mathbb{R})$. Since $f$ is isotopic to the identity the closed one-form $f^{*} a-a$ is exact. Define a cocycle $\iota(a): \operatorname{Ham}(M, d \alpha) \rightarrow C^{\infty}(M) / \mathbb{R}$ by

$$
d(\iota(a)(f)):=f^{*} a-a .
$$

It is straightforward to check that this defines an injective homomorphism

$$
\iota: H^{1}(M ; \mathbb{R}) \rightarrow H^{1}\left(\operatorname{Ham}(M, d \alpha) ; C^{\infty}(M) / \mathbb{R}\right) .
$$

Now we have that

$$
d\left(\mathcal{K}_{\alpha}(f)-\mathcal{K}_{\beta}(f)\right)=f^{*}(\alpha-\beta)-(\alpha-\beta)
$$

which means that $\left[\mathcal{K}_{\alpha}\right]-\left[\mathcal{K}_{\beta}\right]=\iota[\alpha-\beta]$ as claimed.

Remark 2.7. In the definition of $\iota$ we have only used the fact that $f$ acts trivially on the first cohomology of $M$. Thus $\iota$ factors through $H^{1}\left(\operatorname{Diff}\left(M, H^{1}\right) ; C^{\infty}(M) / \mathbb{R}\right)$ the cohomology of the group of diffeomorphisms acting trivially on $H^{1}(M ; \mathbb{R})$.

Proposition 2.8. Let $f \in \operatorname{Ham}(M, d \alpha)$ be a Hamiltonian diffeomorphism generated by the Hamiltonian function $F_{t}: M \rightarrow \mathbb{R}$. Let $x, y \in M$. Then

$\mathcal{K}_{\alpha}(f)(x)-\mathcal{K}_{\alpha}(f)(y)=\int_{f_{t}(x)} \alpha+\int_{0}^{1} F_{t}\left(f_{t}(x)\right) d t-\left(\int_{f_{t}(y)} \alpha+\int_{0}^{1} F_{t}\left(f_{t}(y)\right) d t\right)$,

where $f_{t}$ is the isotopy from the identity to $f$ generated by $F_{t}$. 
Proof. Let $\gamma:[0,1] \rightarrow M$ be a curve from $x$ to $y$ and let $D$ be a singular disc with boundary $\partial D=\gamma+f_{t}(y)-f(\gamma)-f_{t}(x)$.

$$
\begin{aligned}
\mathcal{K}_{\alpha}(f)(x) & -\mathcal{K}_{\alpha}(f)(y) \\
& =-\int_{\gamma} d \mathcal{K}_{\alpha} \\
& =-\int_{\gamma} f^{*} \alpha-\alpha=\int_{\gamma} \alpha-\int_{f(\gamma)} \alpha \\
& =\int_{D} d \alpha-\int_{f_{t}(y)} \alpha+\int_{f_{t}(x)} \alpha \\
& =\int_{0}^{1} \int_{0}^{1} d \alpha\left(d f_{t}(\dot{\gamma}(s)), \dot{f}_{t}(\gamma(s))\right) d t d s-\int_{f_{t}(y)} \alpha+\int_{f_{t}(x)} \alpha \\
& =\int_{0}^{1} \int_{0}^{1}-d F_{t}\left(d f_{t}(\dot{\gamma}(s))\right) d t d s-\int_{f_{t}(y)} \alpha+\int_{f_{t}(x)} \alpha \\
& =-\int_{0}^{1}\left(F_{t}\left(f_{t}(y)\right)-F_{t}\left(f_{t}(x)\right)\right) d t-\int_{f_{t}(y)} \alpha+\int_{f_{t}(x)} \alpha \\
& =\int_{f_{t}(x)} \alpha+\int_{0}^{1} F_{t}\left(f_{t}(x)\right) d t-\left(\int_{f_{t}(y)} \alpha+\int_{0}^{1} F_{t}\left(f_{t}(y)\right) d t\right)
\end{aligned}
$$

Remark 2.9. If $x \in M$ is a fixed point of $f$ and the orbit $f_{t}(x)$ is contractible then $\mathcal{K}_{\alpha}(f)$ is equal to the value of the action functional $\mathcal{A}(F, x)$ up to the above constant (see McDuff-Salamon [16, Section 9] for the background about the action functional).

2.10. Normalization. The restriction of the above cocycle to the subgroup of compactly supported Hamiltonian diffeomorphisms takes values in the space of smooth functions, after choosing $\mathcal{K}_{\alpha}(f)$ so that it has compact support. To see that this is possible we first observe that the function $\mathcal{K}_{\alpha}(f)$ is locally constant outside the support of $f$. Let $x, y \in M$ be two points outside the support of the Hamiltonian function $F_{t}$ generating $f$. Notice that, by definition, a compactly supported Hamiltonian diffeomorphism is generated by a compactly supported Hamiltonian function. According to the formula in Proposition 2.8, we get that $\mathcal{K}_{\alpha}(f)(x)=\mathcal{K}_{\alpha}(f)(y)$ and hence $\mathcal{K}_{\alpha}(f)$ is actually constant outside the support of $f$ so we can normalize it to be compactly supported.

Since the integration with respect to the symplectic volume is a morphism of $\operatorname{Ham}_{c}(M, d \alpha)$-modules $C_{c}^{\infty}(M) \rightarrow \mathbb{R}$, the integral integral $\int_{M} \mathcal{K}_{\alpha}(f)(d \alpha)^{n}$ for normalized $\mathcal{K}_{\alpha}(f)$ is a cocycle in a trivial 
module thus a homomorphism

$$
\text { Cal: } \operatorname{Ham}_{c}(M, d \alpha) \rightarrow \mathbb{R}
$$

called the Calabi homomorphism. Its value can be calculated using the following formula

$$
\int_{M} \mathcal{K}_{\alpha}(f) \omega^{n}=(n+1) \int_{0}^{1} \int_{M} F_{t} \omega^{n} d t
$$

(see Arnold-Khesin [1, Theorem 8.7 on page 247]). Here $F_{t}$ is any Hamiltonian function generating $f$.

On the other hand, if we only assume that a symplectomorphism has a compact support, but not that it is generated by a (compactly supported) Hamitonian flow then some Dehn twists $\tau$ of $T^{*} S^{1}$ supported near the zero section induce $\mathcal{K}_{p d q}(\tau)$ which cannot be normalized to have a compact support. More precisely, let $\tau: T^{*} S^{1} \rightarrow T^{*} S^{1}$ be given by $\tau(p, q)=(p, q+t(p))$, where $0 \leq t(p) \leq 2 \pi, t(p)=0$ for $p \leq-1$ and $t(p)=2 \pi$ for $p \geq 1$. Then $d \mathcal{K}_{p d q}(\tau)=t^{\prime}(p) p d p$ and

$$
\begin{aligned}
\mathcal{K}_{p d q}(\tau)(1, q)-\mathcal{K}_{p d q}(\tau)(-1, q) & =\int_{-1}^{1} t^{\prime}(p) p d p \\
& =\left.t(p) p\right|_{-1} ^{1}-\int_{-1}^{1} t(p) d p \\
& =2 \pi-\int_{-1}^{1} t(p) d p .
\end{aligned}
$$

Thus $\mathcal{K}_{p d q}(\tau)$ has compact support if and only if $\int_{-1}^{1} t(p) d p=2 \pi$.

2.11. The case of a closed manifold. Let $p: \widetilde{M} \rightarrow M$ be the universal covering. Let $G$ be a path connected group of homeomorphisms of $M$ and let

$$
\mathrm{ev}_{x}: G \rightarrow M
$$

be the evaluation map defined by

$$
\mathrm{ev}_{x}(f):=f(x)
$$

where $x \in M$ is a chosen point.

Proposition 2.12 (cf. [12, Remark 1.5.B]). Let $G$ be a path connected group of homeomorphisms of $M$. Suppose that the evaluation map induces the trivial homomorphism on the fundamental group. Then there exists a homomorphism

$$
\Psi: G \rightarrow \operatorname{Homeo}(\tilde{M})
$$


such that $\Psi(f) \circ \pi=\Psi(f)$, where $\pi \in \pi_{1}(M)$ is a deck transformation. Moreover it satisfies $p \circ \Psi(f)=f \circ p$.

Proof. Let $f_{t} \in G$ be an isotopy from the identity to $f$. Define

$$
\Psi(f)(x):=\tilde{f}_{1}(x)
$$

where $\tilde{f}_{t}$ is the lift of the path $f_{t}(p(x))$ starting at $x \in \tilde{M}$.

We have to show that $\Psi(f)$ is well defined. Let $f_{t}^{\prime} \in G(M, \omega)$ be another isotopy from the identity to $f$. Then we get that $\tilde{f}_{1}(x)=$ $\pi\left(\tilde{f}_{1}^{\prime}(x)\right)$, where $\pi:=\left[f_{t}(x) * \overline{f_{t}^{\prime}(x)}\right] \in \pi_{1}(M)$ is the deck transformation induced by the loop $\left(f_{t} * \overline{f_{t}^{\prime}}\right)(x)=e v_{x}\left(f_{t} * \bar{f}_{t}^{\prime}\right)$. Since the evaluation map induces the trivial homomorphism on the fundamental groups, the above element $\pi$ is trivial. Hence $\Psi(f)(x)$ is well defined and clearly it is a lift of $f$.

\section{Example 2.13.}

(1) It is easy to prove that the image of the homomorphism

$$
\left(\mathrm{ev}_{x}\right)_{*}: \pi_{1}(G) \rightarrow \pi_{1}(M)
$$

is contained in the centre of the fundamental group of $M$. Hence, the above proposition applies to any space whose fundamental group has trivial centre.

(2) If $G \subseteq \operatorname{Symp}_{0}(M, \omega)$ and the evaluation map ev $: G \rightarrow M$ induces the trivial map on the fundamental then the homomorphism $\Psi$ takes values in the group $\operatorname{Symp}_{0}\left(\widetilde{M}, p^{*} \omega\right)$.

It is well known (and difficult to prove, see Corollary 9.1.2 in McDuff-Salamon [16]) that the evaluation map on the group of Hamiltonian diffeomorphisms of a closed manifold induces the trivial map on the fundamental group. Hence we get the lifing homomorphism $\Psi: \operatorname{Ham}(M, \omega) \rightarrow \operatorname{Ham}\left(\widetilde{M}, p^{*} \omega\right)$. If $(M, \omega)$ is closed symplectically aspherical then taking the composition $\mathcal{K}_{\alpha} \circ \Psi$ we get a cocyle

$$
\Psi^{*}\left(\mathcal{K}_{\alpha}\right): \operatorname{Ham}(M, \omega) \rightarrow C^{\infty}(\tilde{M}) / \mathbb{R}
$$

It follows from the next proposition that for symplectically hyperbolic manifolds the above cocycle is defined on the group of all symplectomorphisms isotopic with the identity.

Proposition 2.14. Let $(M, \omega)$ be a closed symplectically hyperbolic manifold. Then the evaluation map ev $: \operatorname{Symp}_{0}(M, \omega) \rightarrow M$ induces the trivial homomorphism on the fundamental group. Consequently, there exists a lifting homomorphism $\Psi: \operatorname{Symp}_{0}(M, \omega) \rightarrow \operatorname{Symp}_{0}\left(\tilde{M}, p^{*} \omega\right)$. 
Proof. If $(M, \omega)$ is symplectically hyperbolic then the symplectic form vanishes on tori. That is $s^{*}[\omega]=0$ for any smooth map $s: T^{2} \rightarrow M$. The proof of this fact is not difficult and can be found in Gromov [7, Example 0.2. $A^{\prime}$ ] or Kędra [11, Proposition 1.9]. This implies that the flux homomorphism Flux: $\pi_{1}\left(\operatorname{Symp}_{0}(M, \omega)\right) \rightarrow$ $H^{1}(M ; \mathbb{R})$ is trivial. Consequently the inclusion $\operatorname{Ham}(M, \omega) \rightarrow$ $\operatorname{Symp}_{0}(M, \omega)$ is a homotopy equivalence and the statement follows form the analogous statement for $\operatorname{Ham}(M, \omega)$.

Corollary 2.15. If $(M, \omega)$ is a closed symplectically hyperbolic manifold whose fundamental group has torsion-free centre then there is a well defined cocycle

$$
\Psi^{*}\left(\mathcal{K}_{\alpha}\right): \operatorname{Symp}_{0}(M, \omega) \rightarrow C^{\infty}(\tilde{M}) / \mathbb{R} .
$$

\section{The Polterovich homomorphism}

Let $\operatorname{Ham}(M, \omega, x, y)$ denote the group of Hamiltonian diffeomorphisms fixing two points $x, y \in M$. Let $\gamma:[0,1] \rightarrow M$ be a curve from $x$ to $y$. That is $\gamma(0)=x$ and $\gamma(1)=y$. Let $f \in \operatorname{Ham}(M, \omega, x, y)$. Let $D \subset M$ be a disc whose boundary is the loop given by the concatenation of $\gamma$ and $f(\gamma)$. In other words, let $D: D^{2} \rightarrow M$ be a 2-chain with $\partial D=\gamma-f(\gamma)$. Define the map

$$
\begin{gathered}
\mathcal{P}_{x, y}: \operatorname{Ham}(M, \omega, x, y) \rightarrow \mathbb{R} \\
\mathcal{P}_{x, y}(f):=\int_{D} \omega .
\end{gathered}
$$

Proposition 3.1. Let $(M, \omega)$ be a symplectically aspherical manifold.

(I) If $(M, \omega)$ is exact then the map $\mathcal{P}_{x, y}$ is a well defined homomorphism.

(2) If $M$ is closed then the map $\mathcal{P}_{x, y}$ is a well defined homomorphism on the group $\operatorname{Ham}(M, \omega, x, y)_{\text {cont }}$ consisting of those Hamiltonian diffeomorphisms for which both $x$ and $y$ are contractible fixed points.

In both cases we shall call it the Polterovich homomorphism (cf. Section 2.1 in Polterovich [17]).

A fixed point $x \in M$ of $f$ is called contractible if there exists an isotopy $f_{t}$ from the identity to $f$ such that the loop $f_{t}(x)$ is contractible in $M$. 
3.2. A digression on contractible fixed points. There is an obvious inclusion $\operatorname{Ham}(M, \omega, x, y)_{0} \subset \operatorname{Ham}(M, \omega, x, y)_{\text {cont. }}$ If $\operatorname{dim} M \geq 4$ the above inclusion is an equality. To see this, consider the evaluation fibration

$$
\operatorname{Ham}(M, \omega, x, y) \rightarrow \operatorname{Ham}(M, \omega) \stackrel{\mathrm{ev}_{x, y}}{\longrightarrow} M \times M-\Delta,
$$

where $\operatorname{ev}_{x, y}(f)=(f(x), f(y))$. If both $f_{t}(x)$ and $f_{t}(y)$ are contractible in $M$ then the loop $\left(f_{t}(x), f_{t}(y)\right)$ is contractible in $M \times M-\Delta$ because the diagonal has codimension at least four. Lifting the contraction from $\left(f_{t}(x), f_{t}(y)\right)$ to $(x, y)$ we get an isotopy from the identity to $f$ fixing $x$ and $y$ as claimed.

If $\operatorname{dim} M=2$ then it is easy to construct a Hamiltonian diffeomorphism $f$ of a surface with two contractible fixed points such that any isotopy from the identity to $f$ does not preserve them.

3.3. Back to the proof of Proposition 3.1. We start with the following lemma which is of independent interest. Let $x, y \in M$ and let $(x-y): C^{\infty}(M) / \mathbb{R} \rightarrow \mathbb{R}$ denote the evaluation homomorphism. That is $(x-y)(F):=F(x)-F(y)$.

Lemma 3.4. Let $(M, d \alpha)$ be an exact symplectic manifold. The following diagram is commutative.

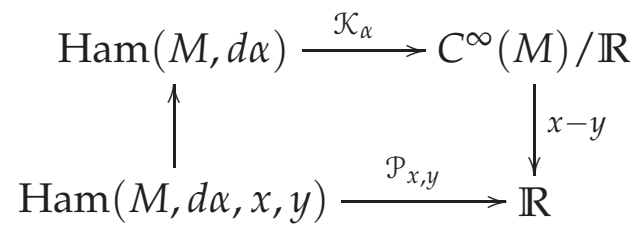

Proof. This is an application of the Stokes Lemma. Let $D$ be a singular disc as in the previous section.

$$
\begin{aligned}
\mathcal{P}_{x, y}(f) & =\int_{D} d \alpha=\int_{\partial D} \alpha \\
& =\int_{\gamma} \alpha-\int_{f(\gamma)} \alpha=\int_{\gamma} \alpha-f^{*} \alpha \\
& =-\int_{\gamma} d \mathcal{K}_{\alpha}(f)=-\int_{\partial \gamma} \mathcal{K}_{\alpha}(f) \\
& =\mathcal{K}_{\alpha}(f)(x)-\mathcal{K}_{\alpha}(f)(y) .
\end{aligned}
$$

Proof of Proposition 3.1 Since $(x-y): C^{\infty}(M) / \mathbb{R} \rightarrow \mathbb{R}$ is a morphism of $\operatorname{Ham}(M, \omega, x, y)$-modules, the composition

$$
(x-y) \circ K_{\alpha}: \operatorname{Ham}(M, d \alpha, x, y) \rightarrow \mathbb{R}
$$


is a cocycle in the trivial module, hence a homomorphism. This proves the proposition in the case of an exact manifold.

In the closed case, let $\tilde{x}, \tilde{y} \in \widetilde{M}$ be points in the preimage $p^{-1}(x)$ and $p^{-1}(y)$ respectively. As in Proposition 2.12 and due to the contractibility of the fixed points, we have a homomorphism

$$
\Psi: \operatorname{Ham}(M, \omega, x, y)_{\text {cont }} \rightarrow \operatorname{Symp}(\tilde{M}, d \alpha, \tilde{x}, \tilde{y}) .
$$

Clearly, the composition $(\tilde{x}-\tilde{y}) \circ \mathcal{K}_{\alpha} \circ \Psi$ is a homomorphism equal to $\mathcal{P}_{x, y}$.

\section{The NON-TRiviality of $\mathcal{K}$}

Theorem 4.1 (Frauenfelder-Polterovich-Schlenk-Schwarz). Let $(M, \omega)$ be a symplectic manifold which is either

(1) closed symplectically aspherical or

(2) exact and convex.

Let $f \in \operatorname{Ham}_{c}(M, \omega)$ be a compactly supported Hamiltonian diffeomorphism. There exist two contractible points $x, y \in M$ such that $\mathcal{P}_{x, y}(f) \neq 0$.

Proof. First observe that, according to Remark 2.9, we have

$$
\mathcal{P}_{x, y}(f)=\mathcal{K}_{\alpha}(f)(x)-\mathcal{K}_{\alpha}(f)(y)=\mathcal{A}(F, x)-\mathcal{A}(F, y),
$$

for any two contractible fixed points $x, y \in M$. This observation has been made by Polterovich in [17].

The second step is to prove that for any Hamiltonian diffeomorphism $f \in \operatorname{Ham}(M, \omega)$ there exist two contractible fixed points $x, y \in M$ such that $\mathcal{A}(F, x)<\mathcal{A}(F, y)$. In the case of a closed symplectically aspherical manifold this was done by Schwarz in [18]. We also refer to McDuff-Salamon (Theorem 9.1.6 on page 283 in [16]) for the proof.

The case of an exact and convex symplectic manifold was done by Frauenfelder and Schlenk [4] based on the ideas of Schwarz. More precisely they define (Section 7 of [4]) a map

$$
c: \operatorname{Ham}_{c}(M, \omega) \rightarrow \mathbb{R}
$$

and they prove (Theorem $7 \cdot 3$ in [4]) that for any compactly supported Hamiltonian diffeomorphism $f$ the number (called the Schwarz norm of $f$ )

$$
c(f)+c\left(f^{-1}\right)
$$

is positive if and only if $f \neq$ ID. Moreover, it follows from Proposition 5.1 and Corollary 6.3 in [4] that there exist two fixed points $x, y \in M$ contractible with respect to the isotopy $f_{t}$ generated by $F$ 
such that $c(f)=\mathcal{A}(F, x)$ and $c\left(f^{-1}\right)=-\mathcal{A}(F, y)$. Finally we get that

$$
0<c(f)+c\left(f^{-1}\right)=\mathcal{A}(F, x)-\mathcal{A}(F, y)=\mathcal{P}_{x, y}(f),
$$

as claimed.

Corollary 4.2. Let $(M, \omega)$ be an exact and symplectic manifold convex at infinity. Then the cohomology class $\left[\mathcal{K}_{\alpha}\right] \in H^{1}\left(\operatorname{Ham}_{c}(M, \omega) ; C_{c}^{\infty}(M)\right)$ is non-zero.

Proof. Let $f \in \operatorname{Ham}_{\mathcal{C}}(M, \omega, x, y)$ be such that $\mathcal{P}_{x, y}(f) \neq 0$. Let

$$
i: \operatorname{Ham}_{c}(M, \omega, x, y) \rightarrow \operatorname{Ham}_{c}(M, \omega)
$$

be the inclusion. Consider the composition

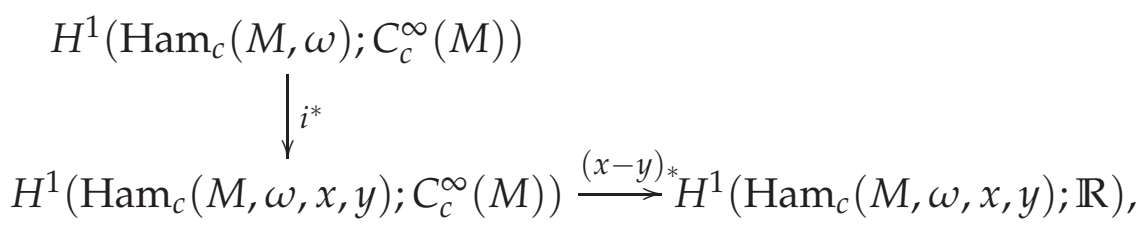

where the second map is induced by the morphism $x-y: C_{c}^{\infty}(M) \rightarrow$ $\mathbb{R}$ of $\operatorname{Ham}_{c}(M, \omega, x, y)$-modules.

Notice that $\mathbb{R}$ is the trivial module hence $H^{1}\left(\operatorname{Ham}_{\mathcal{c}}(M, \omega, x, y) ; \mathbb{R}\right)=$ $\operatorname{Hom}\left(\operatorname{Ham}_{\mathcal{C}}(M, \omega, x, y), \mathbb{R}\right)$. Clearly the class $(x-y)_{*} i^{*}[\mathcal{K}]=\left[\mathcal{P}_{x, y}\right]$ is non-trivial since $\mathcal{P}_{x, y}(f) \neq 0$. Thus the class $[\mathcal{K}] \neq 0$ as claimed.

An analogous proof works in the closed case, so we get the following.

Corollary 4.3. Let $(M, \omega)$ be a closed symplectically aspherical manifold. Then the cohomology class $\Psi^{*}\left[\mathcal{K}_{\alpha}\right] \in H^{1}\left(\operatorname{Ham}(M, \omega) ; C^{\infty}(\widetilde{M}) / \mathbb{R}\right)$ is non-zero.

\section{Distortion OF CyCLIC SUbGROUPS}

5.1. Abstract nonsense. Let $\Gamma$ be a finitely generated group equipped with a word norm $|\cdot|$ and let $(V,\|\cdot\|)$ be a $\Gamma$-module with a norm preserved by the action. Let $\langle f\rangle \subset \Gamma$ be a cyclic group generated by Id $\neq f \in \Gamma$. Consider the following commutative diagram

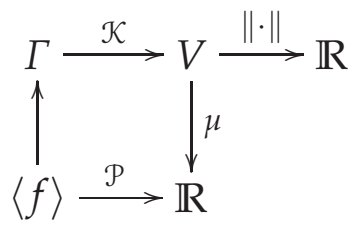


where the maps $\mathcal{K}$ and $\mathcal{P}$ are cocycles, $\mu: V \rightarrow \mathbb{R}$ is a map of $\langle f\rangle$ modules and $\mathbb{R}$ is a trivial $\langle f\rangle$-module.

Lemma 5.2. Suppose that the following conditions hold:

(1) $\left|\mathcal{P}\left(f^{k}\right)\right| \leq\left\|\mathcal{K}\left(f^{k}\right)\right\|$ for all $k \in \mathbb{Z}$,

(2) $\mathcal{P}$ is a nontrivial homomorphism,

Then $\langle f\rangle$ is undistorted in $\Gamma$.

Proof. First observe that any cocycle $\mathcal{K}: \Gamma \rightarrow V$ is Lipschitz. To see this, let $g_{1}, \ldots, g_{k} \in \Gamma$ be the generators inducing the norm $|\cdot|$. Let $m:=\max \left\{\left\|\mathcal{K}\left(g_{i}\right)\right\| \mid i=1, \ldots, k\right\}$. If $f=g_{i_{1}} \cdot \ldots \cdot g_{i_{|f|}}$ then we get that

$$
\begin{aligned}
\|\mathcal{K}(f)\| & =\left\|\mathcal{K}\left(g_{i_{1}} \cdot \ldots \cdot g_{i_{|f|} \mid}\right)\right\| \\
& =\left\|\sum_{j=1}^{|f|} \mathcal{K}\left(g_{i_{j}}\right) \cdot g_{i_{j+1}} \cdot \ldots \cdot g_{i_{|f|} \mid}\right\| \\
& \leq \sum_{j=1}^{|f|}\left\|\mathcal{K}\left(g_{i_{j}}\right)\right\| \leq m \cdot|f| .
\end{aligned}
$$

Let Id $\neq f \in \Gamma$. The hypothesis together with the above observation immediately imply the following inequalities:

$$
0<n|\mathcal{P}(f)|=\left|\mathcal{P}\left(f^{n}\right)\right| \leq\left\|\mathcal{K}\left(f^{n}\right)\right\| \leq m \cdot\left|f^{n}\right| .
$$

It follows that

$$
\left|f^{n}\right| \geq \frac{|\mathcal{P}(f)|}{m} \cdot n>0,
$$

which finishes the proof.

5.3. Proof of Theorem 1.5. Recall that $(M, d \alpha)$ is an exact and convex symplectic manifold and $\Gamma \rightarrow \operatorname{Ham}_{\mathcal{c}}(M, d \alpha)$ is an effective action of a finitely generated group. Let $\operatorname{Id} \neq f \in \Gamma$. We shall show that the cyclic subgroup $\langle f\rangle \subset \Gamma$ is undistorted.

Let the oscillation osc: $C_{c}^{\infty}(M) \rightarrow \mathbb{R}$ be defined by $\operatorname{osc}(F):=$ $\max _{x \in M} F-\min _{x \in M} F$. It is a norm on the space of compactly supported functions. We have the following commutative diagram

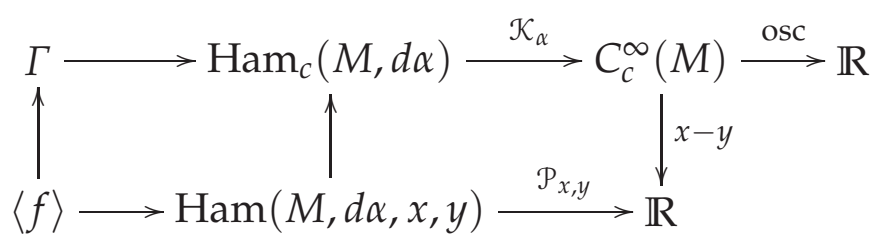


Observe that the inequality $\mathcal{P}_{x, y}(f) \leq \operatorname{osc}_{\mathcal{K}}(f)$ is trivially satisfied since $\mathcal{P}_{x, y}(f)=\mathcal{K}_{\alpha}(f)(x)-\mathcal{K}_{\alpha}(f)(y)$, according to Lemma 3.4 The points $x, y \in M$ are chosen so that the Polterovich homomorphism is nontrivial $\mathcal{P}_{x, y}(f)>0$. Hence we are precisely in the situation when the hypothesis of Lemma 5.2 is satisfied. Thus applying this lemma finishes the proof.

5.4. An example of a compactly supported action with distorted cyclic subgroups. This section is essentially due to Łukasz Grabowski. If $\Gamma$ acts on a manifold $M$ with a global fixed point $x$ and the isotropy representation can be deformed to the trivial one. This means that the isotropy representation lies in the connected component of the trivial one inside the representation variety $\{\Gamma \rightarrow$ $\left.\mathrm{GL}\left(T_{x} M\right)\right\}$. Then one can replace $x$ by a disc and extend the action of $\Gamma$ to an action which is trivial in some (smaller) disc. In other words one can produce a compactly supported action of $\Gamma$ on $M-\{x\}$ which has essentially the same orbit structure.

Consider the projective action of the Heisenberg group $H:=$ $N(3, \mathbb{Z})$ of uppertriangular matrices with integer entries on two dimensional sphere $S^{2}$. The isotropy representation on the tangent space to the north pole $(1,0,0)$ is given by

$$
\left[\begin{array}{lll}
1 & a & c \\
0 & 1 & b \\
0 & 0 & 1
\end{array}\right] \mapsto\left[\begin{array}{ll}
1 & b \\
0 & 1
\end{array}\right]
$$

and it clearly can be deformed to the trivial one. Thus we get that the Heisenberg group acts smoothly with compact supports on an open ball $S^{2}-\{x\}$. This can be generalized in an obvious way to higher dimensions. The centre of the Heisenberg group is a distorted cyclic subgroup as it can be directly calculated (see also Gromov [8, page 52]). We have then proved the following result.

Proposition 5.5. There exists a smooth, compactly supported action of the Heisenberg group $H$ on $\mathbb{R}^{n}$ for any $n \geq 2$.

\section{A proof of the Polterovich theorem}

Proposition 6.1. Let $(M, \omega)$ be a closed symplectically hyperbolic manifold. Then the composition

$$
\operatorname{Ham}(M, \omega) \stackrel{\Psi}{\rightarrow} \operatorname{Ham}(\widetilde{M}, d \alpha) \stackrel{\mathcal{K}_{\alpha}}{\rightarrow} C^{\infty}(\widetilde{M}) / \mathbb{R}
$$

takes values in $C_{b}^{\infty}(\tilde{M}) / \mathbb{R}$, where $C_{b}^{\infty}(\tilde{M})$ denotes the space of bounded functions. 
Proof. Let $f \in \operatorname{Ham}(M, \omega)$ and let $x, y \in \widetilde{M}$ be any two points. Let $f_{t}$ be an isotopy form the identity to $f$ generated by a Hamiltonian function $F_{t}: M \rightarrow \mathbb{R}$ and let $\tilde{f}_{t}$ and $\tilde{F}_{t}$ be the corresponding lifts to $\widetilde{M}$. According to Proposition 2.8 we get the first equality in the following calculation.

$$
\begin{aligned}
& \left|\mathcal{K}_{\alpha}(\Psi(f))(x)-\mathcal{K}_{\alpha}(\Psi(f))(y)\right| \\
= & \left|\int_{\tilde{f}_{t}(x)} \alpha+\int_{0}^{1} \tilde{F}_{t}\left(\tilde{f}_{t}(x)\right) d t-\int_{\tilde{f}_{t}(y)} \alpha+\int_{0}^{1} \tilde{F}_{t}\left(\tilde{f}_{t}(y)\right) d t\right| \\
\leq & 2 \cdot C \cdot \max _{x} \operatorname{Length}\left(\tilde{f}_{t}(x)\right)+2 \cdot \max _{x, t} \tilde{F}_{t}(x)
\end{aligned}
$$

The last quantity is finite because $\tilde{F}_{t}$ and $\tilde{f}_{t}$ are lifts of $F_{t}$ and $f_{t}$ respectively and the latter are defined on a compact manifold. Also, the length is calculated with respect to the metric induced from $M$. We used the easy fact that

$$
\int_{f_{t}(x)} \alpha \leq C \cdot \text { Length }\left(f_{t}(x)\right),
$$

where $C>0$ is the constant bounding the norm of $\alpha$.

Let $\Gamma \rightarrow \operatorname{Ham}(M, \omega)$ be an effective action of a finitely generated group. We shall show that every nontrivial cyclic subgroup $\langle f\rangle$ of $\Gamma$ is undistorted.

Proof of Theorem [.8, Let $g_{1}, g_{2}, \ldots, g_{k} \in \Gamma$ be generators and let $m:=\max _{i} \mathcal{K}_{\alpha}\left(\Psi\left(g_{i}\right)\right)$ be the Lipschitz constant (cf. Lemma [5.2). Due to the above proposition, $m$ is finite and we have the following estimate

$$
\left|f^{n}\right| \geq \frac{\operatorname{osc}\left(\mathcal{K}_{\alpha}\left(\Psi\left(f^{n}\right)\right)\right)}{m} \geq \frac{\mathcal{P}_{x, y}\left(f^{n}\right)}{m}=n \frac{\mathcal{P}_{x, y}(f)}{m}>0 .
$$

The existence of fixed points $x, y \in M$ such that $\mathcal{P}_{x, y}>0$ is ensured by Theorem 4.1 .

\section{THE RELATION WITH THE FLUX HOMOMORPHISM}

Let $p: \widetilde{M} \rightarrow M$ be the universal cover of a closed manifold. Let $C_{b}^{\infty}(\widetilde{M})$ denote the space of bounded functions and let

$$
\Phi: H^{1}(M ; \mathbb{R}) \rightarrow C^{\infty}(\tilde{M}) / C_{b}^{\infty}(\widetilde{M})
$$

be a homomorphism defined by $d \Phi[a]=p^{*} a$. It is easy to check that this homomorphism is injective. 
Proposition 7.1. Let $(M, \omega)$ be a closed symplectically hyperbolic manifold. The following diagram is commutative.

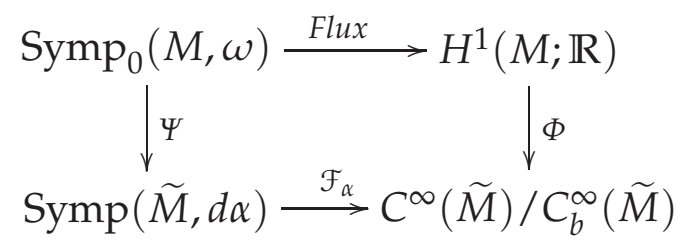

Here $\mathcal{F}_{\alpha}$ is the composition of $\mathcal{K}_{\alpha}$ with the natural projection.

Proof. We shall show that $\Phi(\operatorname{Flux}(f))$ equals $\mathcal{K}_{\alpha}(\Psi(f))$ up to a bounded summand. Notice that the homomorphism $\Psi: \operatorname{Symp}_{0}(M, \omega) \rightarrow$ $\operatorname{Symp}_{0}\left(\tilde{M}, p^{*} \omega\right)$ exists due to Proposition 2.14. If $f_{t}$ is an isotopy from the identity to $f$ then

$$
\int_{0}^{1}\left(\iota_{\dot{f}_{t}} \omega\right) d t \in \Omega^{1}(M)
$$

is a differential form representing the flux of $f$. In the following calculation let $X_{t}:=\frac{d}{d t} \Psi\left(f_{t}\right)$.

$$
\begin{aligned}
d \Phi(\operatorname{Flux}(f)) & =p^{*}\left(\int_{0}^{1}\left(\iota_{\dot{f}_{t}} \omega\right) d t\right) \\
& =\int_{0}^{1}\left(\iota x_{t} d \alpha\right) d t \\
& =\int_{0}^{1}\left(\iota_{X_{t}} \Psi\left(f_{t}\right)^{*} d \alpha\right) d t \\
& =\int_{0}^{1}\left(\mathcal{L}_{X_{t}} \Psi\left(f_{t}\right)^{*} \alpha-d \iota_{X_{t}} \Psi\left(f_{t}\right)^{*} \alpha\right) d t \\
& =\Psi(f)^{*} \alpha-\alpha-d \int_{0}^{1} \Psi\left(f_{t}\right)^{*} \alpha\left(\mathcal{X}_{t}\right) d t \\
& =d\left(\mathcal{K}_{\alpha}(\Psi(f))-\int_{0}^{1} \Psi\left(f_{t}\right)^{*} \alpha\left(X_{t}\right) d t\right)
\end{aligned}
$$

Further we have that

$$
\left|\int_{0}^{1} \Psi\left(f_{t}\right)^{*} \alpha\left(X_{t}\right) d t\right| \leq C \cdot \max _{(x, t) \in M \times[0,1]}\left\|D_{x} \Psi\left(f_{t}\right)\right\| \cdot \text { Length }\left(f_{t}(x)\right),
$$

where $C$ is a constant bounding the norm of the primitive $\alpha$. Clearly, the right hand side of the above inequality is finite. This finishes the proof. 
As a byproduct of the above result we get the following characterization of Hamiltonian diffeomorphisms of a closed symplectically hyperbolic manifold.

Corollary 7.2. Let $(M, \omega)$ be a closed symplectically hyperbolic manifold. A symplectic diffeomorphism $f \in \operatorname{Symp}_{0}(M, \omega)$ is Hamiltonian if and only if $\mathcal{K}_{\alpha}(\Psi(f))$ is bounded.

\section{REFERENCES}

[1] V. I. Arnold et B. A. KHesin - Topological methods in hydrodynamics, Applied Mathematical Sciences, vol. 125, Springer-Verlag, New York, 1998.

[2] J. FRANKS - "Distortion in groups of circle and surface diffeomorphisms", Dynamique des difféomorphismes conservatifs des surfaces: un point de vue topologique, Panor. Synthèses, vol. 21, Soc. Math. France, Paris, 2006, p. 35-52.

[3] U. FrauenfELder et F. SCHLENK - "Slow entropy and symplectomorphisms of cotangent bundles", arXiv:math/0404017 (2004).

[4] _ "Hamiltonian dynamics on convex symplectic manifolds", Israel J. Math. 159 (2007), p. 1-56.

[5] J.-M. Gambaudo et É. Ghys - "Enlacements asymptotiques", Topology 36 (1997), no. 6, p. 1355-1379.

[6] R. E. GompF - "Symplectically aspherical manifolds with nontrivial $\pi_{2}$ ", Math. Res. Lett. 5 (1998), no. 5, p. 599-603.

[7] M. Gromov - "Kähler hyperbolicity and $L_{2}$-Hodge theory", J. Differential Geom. 33 (1991), no. 1, p. 263-292.

[8] _ , "Asymptotic invariants of infinite groups", Geometric group theory, Vol. 2 (Sussex, 1991), London Math. Soc. Lecture Note Ser., vol. 182, Cambridge Univ. Press, Cambridge, 1993, p. 1-295.

[9] R. IbÁñez, J. KęDRA, Y. RudyaK et A. Tralle - "On fundamental groups of symplectically aspherical manifolds", Math. Z. 248 (2004), no. 4, p. 805-826.

[10] J. Ketdra, Y. RudyaK et A. Tralle - "On fundamental groups of symplectically aspherical manifolds. II. Abelian groups", Math. Z. 256 (2007), no. 4, p. $825-835$.

[11] J. KęDRA - "Symplectically hyperbolic manifolds", Differential Geom. Appl. (2008, to appear).

[12] F. Lalonde et L. Polterovich - "Symplectic diffeomorphisms as isometries of Hofer's norm", Topology 36 (1997), no. 3, p. 711-727.

[13] A. Lubotzky, S. Mozes et M. S. Raghunathan - "The word and Riemannian metrics on lattices of semisimple groups", Inst. Hautes Études Sci. Publ. Math. (2000), no. 91, p. 5-53 (2001).

[14] D. McDufF - "Enlarging the Hamiltonian group", J. Symplectic Geom. 3 (2005), no. 4, p. 481-530, Conference on Symplectic Topology.

[15] D. McDuff et D. Salamon - Introduction to symplectic topology, second éd., Oxford Mathematical Monographs, The Clarendon Press Oxford University Press, New York, 1998. 
[16] _ J-holomorphic curves and symplectic topology, American Mathematical Society Colloquium Publications, vol. 52, American Mathematical Society, Providence, RI, 2004.

[17] L. Polterovich - "Growth of maps, distortion in groups and symplectic geometry", Invent. Math. $\mathbf{1 5 0}$ (2002), no. 3, p. 655-686.

[18] M. SchwARz - "On the action spectrum for closed symplectically aspherical manifolds", Pacific J. Math. 193 (2000), no. 2, p. 419-461.

Instytut MatematyczNy, UniWeRsytet WrocŁaWSKI, PL. GRUNWALDZKi 2/4, Wroceaw, Poland

Department of Mathematics, The Ohio State University, 10o Math Tower, 231 West 18Th Avenue, Columbus, OH 43210-1174, usa

E-mail address: sgal@math.uni.wroc.pl

URL: http://www.math.uni.wroc.pl/ ${ }^{\text {sgal }}$

Current address: Mathematical Sciences, University of Aberdeen, Meston Building, Aberdeen AB243UE, Scotland, UK

Current address: Institute of Mathematics, University of Szczecin, ul. Wielkopolska 15, 70-451 Szczecin, Poland

E-mail address: kedra@maths.abdn.ac.uk

URL: http://www. maths.abdn.ac.uk/ kedra 